\title{
Maximum rate resource allocation algorithms with multiuser diversity and QoS support for downlink OFDMA based WiMAX system
}

\begin{abstract}
Orthogonal frequency division multiple access (OFDMA) has been adopted as the core physical layer access for a few broadband wireless access networks such as worldwide interoperability for microwave access (WiMAX). In WiMAX the primary concern is quality of service (QoS) support which aims to satisfy the diverse service requirements and to guarantee the required data rates from the available resources. Therefore, designing resource allocation algorithm becomes vital to maximize spectral efficiency. In this paper, two resource allocation algorithms are proposed, namely, weighted-rate adaptive slot allocation (WASA), and feedback delay-based slot allocation (FDSA) for OFDMA downlink system. The aim is to improve system performance by exploiting the available resources in a twodimensional domain, and assigning the capacity for each user based on their achievable data rates and channel responses. During all these processes, fairness for all service types is ensured. Both approaches allocate appropriate resources and provide higher data rates for different service types by employing a weighted-rate factor and feedback information delay which are made to be greater than the minimum QoS requirements. Simulation results indicate that both WASA and FDSA achieve significant performance improvements in terms of spectral efficiency, outage probability, and fairness against the conventional OFDMATDMA and MAX-SNR algorithms. Comparison with recent works developed as adaptive slot allocation and reservation-based slot allocation has also been performed. The performance gains of both our proposed algorithms can be attributed to satisfying of the diverse multiuser and QoS requirements.
\end{abstract}

Keyword: Resource allocation; QoS; Scheduling; OFDMA; WiMAX 